\title{
Um mapeamento sistemático sobre o uso da Lousa Digital Interativa no processo de ensino-aprendizagem
}

\author{
Henrique A. Silva, Diego Barbosa B. Costa, \\ Roberto Goulart S. Filho, Ana Carolina G. Inocêncio \\ Curso de Ciência da Computação - Universidade Federal de Goiás (UFG) \\ Caixa Postal 01 - CEP 75.801 - 615 - Jataí - GO - Brasil \\ \{henriarasilva, diegocostacmp, robertogsfl, anacarolina.inocencio\}@gmail.com
}

\begin{abstract}
This article aims to select and organize, through the collection of information, the difficulties that can be found in the pedagogical use of Interactive Whiteboard (IWB). This mapping intends to list these difficulties, as well methods proposed to improve the use of this technology. In total, 4,219 papers were found. When applying the inclusion and exclusion criteria were selected 36 papers for a thorough reading which led to rejection of 26 and 10 were accepted. With the mapping, it was noticed that in order to improve the teaching-learning process, it is important to plan what activities to use, as well as pedagogical training for the use of IWB.
\end{abstract}

Resumo. Este artigo tem como objetivo a seleção e a organização, por meio da coleta de informações, das dificuldades que podem ser encontradas no uso pedagógico da Lousa Digital Interativa (LDI). Este mapeamento tem por intenção elencar estas dificuldades, bem como métodos propostos para melhoria do uso desta tecnologia. No total, foram encontrados 4.219 trabalhos. Ao aplicar os critérios de inclusão e exclusão foram selecionados 36 trabalhos para uma leitura minuciosa o que levou a rejeição de 26 e 10 foram aceitos. Com o mapeamento, percebeu-se que para melhorar o processo de ensino-aprendizagem, é importante um planejamento de quais atividades utilizar, bem como treinamento pedagógico para o uso da LDI.

\section{Introdução}

A Lousa Digital Interativa (LDI) é uma tecnologia criada por volta de 1991 que é muito poderosa quando se trata do processo de ensino-aprendizagem. Por meio dela é possível obter a atenção e o interesse dos alunos nas aulas, tendo em vista que vivenciam um mundo altamente tecnológico quando estão fora da escola [Ferrari et al. 2016].

Apesar de estarem presentes em diversas escolas, em razão de sua possibilidade incontestável de haver grande interatividade, várias vezes as LDIs não são exploradas pedagogicamente pelos professores. O principal motivo disto é a dificuldade enfrentada durante o uso, tanto no modo de usar quanto o de possuir o conhecimento necessário para tal atividade [Santos et al. 2013] e [Ferrari et al. 2016].

Considerando as dificuldades enfrentadas durante o uso da LDI, este mapeamento tem por intenção identificar os motivos destas dificuldades, bem como listar métodos de resolução das mesmas, com o objetivo de auxiliar os interessados em inserir a LDI no processo de ensino-aprendizagem. 
VI Congresso Brasileiro de Informática na Educação (CBIE 2017)

Anais do XXIII Workshop de Informática na Escola (WIE 2017)

\section{Metodologia}

Para responder as questões de pesquisas da Seção 2.1, este mapeamento sistemático tem como foco analisar e interpretar dados relevantes, como sugere [Keele 2007] para fazer revisão sistemática, em relação ao uso da LDI. Para executar esta atividade, este mapeamento foi dividido de acordo com as quatro etapas estabelecidas por [Petersen et al. 2008]:

1. Definição das questões de pesquisa;

2. Execução da pesquisa por meio de estudos primários relevantes;

3. Extração e síntese de dados;

4. Análise de dados.

\subsection{Questões de Pesquisa}

Busca-se responder: por que, apesar das LDIs já estarem inseridas em muitas escolas, elas ainda são pouco utilizadas nas salas de aula? A partir deste questionamento quatro sub-questões foram levantadas:

1. Quais são os principais benefícios do uso da LDI para a Educação?

2. Quais as maiores dificuldades no uso da LDI?

3. Quais métodos foram propostos para o melhoramento do uso da LDI?

4. Quais formas de avaliação foram utilizadas para verificar o uso da LDI?

\subsection{Pesquisa por meio de Estudos Primários}

A fim de ter uma boa abrangência, por meio da string de busca em motores que retornassem uma boa quantidade de resultados referentes ao assunto tratado neste mapeamento sistemático, a pesquisa foi feita de forma manual nos seguintes motores de busca: Google Acadêmico $^{1}$, Periódicos CAPES ${ }^{2}$, e IEEE Xplore ${ }^{3}$. As palavras-chave para executar a pesquisa foram as seguintes, em português: "lousa digital"; ensino; "objetos de aprendizagem"; "software educacionais"; "jogos educacionais"; usabilidade; melhoria; melhoramento; aprimoramento; critérios; inspeção; heurísticas; diretrizes. E em inglês: "interactive whiteboard"; "learning objects"; "educational softwares"; "educational games"; using; usability; improving; improvement; amelioration; inspection; criteria; heuristics; guidelines.

Pelas palavras-chave, gerou-se a string de busca a partir da combinação mesmas, tanto no idioma inglês quanto no português, da seguinte forma:

- Português: "lousa digital" AND (ensino OR "objetos de aprendizagem" OR "softwares educacionais" OR "jogos educacional") AND (usabilidade OR melhoria OR melhoramento OR aprimoramento OR critérios OR inspeção OR heurísticas OR diretrizes);

- Inglês: ("interactive whiteboard") AND (("learning objects") OR ("educational softwares") OR (“educational games”)) AND (using OR usability OR improving OR improvement OR amelioration OR inspection OR criteria OR heuristics OR guidelines).

\footnotetext{
${ }^{1}$ scholar.google.com.br

${ }^{2}$ www.periodicos.capes.gov.br

${ }^{3}$ ieeexplore.ieee.org
} 
Na seleção de trabalhos, foram definidos os seguintes critérios de inclusão: estar escrito em português ou inglês; ser a versão completa do trabalho; estar disponível na web; versar sobre o cenário atual da utilização da LDI em alguma área de conhecimento (Educação, Biologia, Matemática, etc); ter data de publicação igual ou superior ao ano de 2012; propor método(s) para o melhoramento do uso da LDI; conter uma forma de avaliação de método.

Além dos critérios de exclusão consistirem da negação dos critérios de inclusão, foram adotados os seguintes critérios de exclusão: ser um resumo, não apresentar uma descrição detalhada sobre o problema tratado em questão; ser uma versão mais antiga ou ser duplicado; não dissertar sobre dificuldades/falhas ou melhorias.

\section{Resultados e Discussões}

\subsection{Quais são os principais benefícios do uso da LDI para a Educação?}

- Interatividade: esse fator é unanime para todos os autores, pois é a característica primordial que impulsiona o uso a LDI. De acordo com a literatura ([Aytaç 2013], [Maher et al. 2012], [Whyte et al. 2014] ), a LDI, por ser muito interativa, pode proporcionar a colaboração em grupo, pois os alunos tendem a participar mais (mantendo o foco na aula) [Aytaç 2013], o que geralmente acelera muito o processo de ensino-aprendizagem;

- Facilidade na criação de jogos e/ou brincadeiras para o aprendizado: esse é o aspecto mais interessante na visão pedagógica. Muitos alunos gostam de jogar, interagir, pintar e realizar qualquer outra atividade utilizando a LDI. O touch scheen pode substituir os movimentos do mouse por toques na tela, isso pode ser feito principalmente utilizando Objetos de Aprendizagem (OAs) [Fiscarelli et al. 2016];

- Visual atrativo e/ou curioso: o simples ato do professor dar uma aula normal utilizando os recursos da LDI, de modo que ele faça anotações escritas ou que opere softwares [Maher et al. 2012], mostre vídeos, apresente e edite imagens, e também navegue na Internet, entre várias outras atividades, a forma que o professor conduz essas atividades já desperta a atenção do aluno naturalmente;

- Assemelhação aos recursos tecnológicos: a LDI pode ser usada tanto como um quadro normal com recursos a mais, tanto quanto uma ferramenta pedagógica digital visando o entretenimento no processo de aprendizagem. Uma das vantagens é que suas características são semelhantes as dos recursos tecnológicos do cotidiano, e assim os alunos já se sentem familiarizados, e a adaptação a nova forma de manuseio se torna mais fácil e rápida [Ferrari et al. 2016];

- Recursos permitem adaptação a públicos distintos: apesar da LDI ser muito utilizada para a Educação, ela pode facilmente ser adaptada a qualquer público, basta utilizar de novas ferramentas de auxílio que visem esse novo público, como um OA ou qualquer outra ferramenta educacional, que possa ser encontrada em repositórios online. Evidentemente, os recursos da LDI foram feitos para que o professor tenha liberdade para planejar suas aulas conforme a necessidade [Kalinke 2013] e [Fiscarelli et al. 2016]. Ela pode ser adaptada aos diferentes estilos de aprendizagem, aos diferentes níveis de capacidade e interesse intelectual, às diferentes situações de ensino e aprendizagem, isso dá margem à criação de novas formas de abordagens [Kalinke 2013] e [Whyte et al. 2014]. 
VI Congresso Brasileiro de Informática na Educação (CBIE 2017)

Anais do XXIII Workshop de Informática na Escola (WIE 2017)

\subsection{Quais as maiores dificuldades no uso da LDI?}

- Falta de formação na área: esse fator é o mais determinante para o uso da LDI. Seu uso não é simples, ela dispõe de tantos recursos que as vezes é até um pouco difícil de interpretá-los, mas muitas vezes as atividades da LDI dispõe de uma manual de uso, e muitas vezes é necessário lê-lo [Whyte et al. 2014];

- Resistência/dificuldades dos professores quanto ao se adaptarem/utilizarem utilização de novas tecnologias: esse fator foi relatado como o mais determinante para o desuso da LDI [Whyte et al. 2014] e [Santos et al. 2013];

- Grande diferença no manuseio dos dispositivos comuns: um grande diferencial incontestável da LDI, é a forma em que as atividades são conduzidas. Isso a difere imensamente de qualquer outra tecnologia digital utilizada para o ensino, um fator indiscutível é o seu tamanho, por exemplo [Esteves et al. 2013].

\subsection{Quais métodos foram propostos para o melhoramento do uso da LDI?}

- Plano de Formação para professores: já que a LDI é para público distintos, não existe uma regra geral para todos os casos. O ideal é que o professor adapte as atividades desenvolvidas de acordo com o gosto da turma. A formação dos professores é fundamental nesse ponto vista. $\mathrm{O}$ fato dos professores não serem capacitados para executar tal tarefa dificulta muito o uso da LDI [Freitas 2013];

- Melhor escolha das atividades interativas: essa escolha não é algo trivial. Primeiramente, exigi-se um esforço para definir quais atividades devem ser usadas. Para [Fiscarelli et al. 2016], embora existam repositórios cheios de OAs para se usar, muitos não se adequam a dinâmica da LDI. Isso foi comprovado em seu experimento em que a maioria (55\%) dos OAs testados, exigiam o uso do teclado, esses OAs não eram adequados/adaptáveis a dinâmica da LDI no contexto de uso. Embora muitos dos programas, como softwares educacionais já apresentem um ambiente bem amigável, eles exigem uma análise criteriosa, para que se possa extrair mais seu potencial pedagógico [Freitas 2013] e [Maher et al. 2012].

\subsection{Quais formas de avaliação foram utilizadas para verificar o uso da LDI?}

- Questionário sobre uso respondido pelos próprios participantes: Feito sob orientação de preenchimento [Ferrari et al. 2016];

- Entrevista após o uso da LDI: No trabalho de [Esteves et al. 2013], 95\% dos alunos disseram que gostam de ir a LDI realizar atividades e $62 \%$ deles dizem entender melhor o conteúdo quando o professor realiza atividades com a LDI.

\section{Considerações finais}

A partir dos resultados da análises das questões levantadas na seção 3, percebeu-se que os benefícios do uso da LDI são diversos, pois em todos os trabalhos foram constatados que a LDI melhorou a qualidade do processo de ensino-aprendizagem e despertou uma maior colaboração entre os alunos.

Como se trata de uma tecnologia relativamente nova nas escolas, foram encontradas dificuldades desde o conhecimento de como a LDI funciona, até o conhecimento técnico de como utilizá-la e quais atividades escolher. Muito foi discutido sobre programas de formaçãode docentes e incentivos de uso da LDI. 
VI Congresso Brasileiro de Informática na Educação (CBIE 2017)

Anais do XXIII Workshop de Informática na Escola (WIE 2017)

A resistência às mudanças tecnológicas, muitas vezes, é uma grande dificuldade inicial. Para que o professor consiga utilizar os recursos da LDI, sendo preciso paciência, foco e persistência. Neste sentido, o resultado final do uso desta tecnologia se mostrou satisfatório.

\section{Referências}

Aytaç, T. (2013). Interactive whiteboard factor in education: Students' points of view and their problems. Educational Research and Reviews, 8(20):1907.

Esteves, R. F., Souza, C. B. G. d., and Fiscarelli, S. H. (2013). A lousa digital interativa no ensino fundamental: um estudo de caso de uma escola municipal brasileira. Política e Gestão Educacional, pages 186-197.

Ferrari, D. P. et al. (2016). A lousa digital no processo de alfabetização desafios e possibilidades. Monografia (Especialização em Educação na Cultura Digital), UFSC (Universidade Federal de Santa Catarina), Palhoça, SC.

Fiscarelli, S. H., Morgado, C. L., and Félix, M. A. (2016). Objetos de aprendizagem e lousas digitais interativas: uma proposta de avaliação de objetos de aprendizagem para ensino de matemática. Revista Ibero-Americana de Estudos em Educação, 11(25).

Freitas, A. V. (2013). Formação do professor de matemática envolvendo novos recursos tecnológicos: reflexões e propostas. Brazilian Geographical Journal: Geosciences and Humanities research medium, 4(2 S).

Kalinke, M. A. (2013). Uma experiência com o uso de lousas digitais na formação de professores de matemática. XI ENEM-ENCONTRO NACIONAL DE EDUCAÇÃO MATEMÁTICA.

Keele, S. (2007). Guidelines for performing systematic literature reviews in software engineering. In Technical report, Ver. 2.3 EBSE Technical Report. EBSE. sn.

Maher, D., Phelps, R., Urane, N., and Lee, M. (2012). Primary school teachers' use of digital resources with interactive whiteboards: The australian context. Australasian Journal of Educational Technology, 28(1).

Petersen, K., Feldt, R., Mujtaba, S., and Mattsson, M. (2008). Systematic mapping studies in software engineering. In EASE, volume 8, pages 68-77.

Santos, L. M. A., Varaschini, P. L., and Martins, S. L. M. (2013). Lousa digital: mapeamento de recursos para utilização em sala de aula. Electronic Journal of Management, Education and Environmental Technology (REGET), 15(15):2895-2901.

Whyte, S., Schmid, E. C., van Hazebrouck Thompson, S., and Oberhofer, M. (2014). Open educational resources for call teacher education: the itilt interactive whiteboard project. Computer Assisted Language Learning, 27(2):122-148. 